\title{
Choice of Measurement Locations of Nonlinear Structures Using Proper Orthogonal Modes and Effective Independence Distribution Vector
}

\author{
T. G. Ritto \\ Departamento de Engenharia Mecânica, Centro de Tecnologia, Universidade Federal do Rio de Janeiro, \\ 21945-970 Ilha do Fundão, RJ, Brazil \\ Correspondence should be addressed to T. G. Ritto; tritto@mecanica.ufrj.br
}

Received 26 February 2013; Accepted 20 June 2013; Published 30 January 2014

Academic Editor: Hassan Haddadpour

Copyright ( 2014 T. G. Ritto. This is an open access article distributed under the Creative Commons Attribution License, which permits unrestricted use, distribution, and reproduction in any medium, provided the original work is properly cited.

This paper proposes a methodology to automatically choose the measurement locations of a nonlinear structure/equipment that needs to be monitored while operating. The response of the computational model (or experimental data) is used to construct the proper orthogonal modes applying the proper orthogonal decomposition (POD), and the effective independence distribution vector (EIDV) procedure is employed to eliminate, iteratively, locations that contribute less for the independence of the target proper orthogonal modes.

\section{Introduction}

There are many works discussing the placement of sensors in a dynamical structure. One can use, for instance, observability measure $[1,2]$ to identify the best locations or entropy measure [3]. Debnath et al. [4] did a literature survey of the methods that have been used to identify the optimal location of sensors.

Udwadia and Garba [5] propose to optimally locate sensors with a technique that uncouples the solution of an optimization and an identification problem, and Fisher information matrix is used. Concerning optimal sensor locations for control, [6] use a quadrature formula to obtain the sensor locations, given a feedback control law. In [7], explicit solutions of generalized algebraic Riccati equations are developed, such that optimal sensor locations are obtained for a control strategy.

Guyan [8] proposed a strategy known as Guyan reduction [9] that can be used for choice of sensor locations. This strategy eliminates locations with low inertia forces compared to elastic forces. Qureshi et al. [10] use the design criterion of maximizing the determinant of Fisher information matrix to optimally place sensors in distributed systems. Kammer [11] proposes the effective independence method to select measurement locations for modal identification of large space structures. This method ranks the contribution of each candidate sensor location to the linear independence of the corresponding target modes; it also maximizes the determinant of Fisher information matrix. This method is also known as the effective independence vector distribution (EIVD) [9]. A recent application of this method for mistuning identification of blisks is found in [12]. The effective independence concept was also modified to take into account strain energy [13], a strategy called energy matrix rank optimization (EMRO), and kinetic energy [14], a strategy called energy optimization technique (EOT).

Techniques that use the normal modes of the structures, such as Fisher information matrix, deal with linear structures. As we are interested in nonlinear structures, some extension has to be pursued. Nonlinear normal modes (NNM) $[15,16]$, which can be regarded as nonlinear extensions of the classical normal modes, could be applied in this attempt. However, in the present paper, we will pursue another strategy and not use the NNM because they can be difficult to compute, they usually disregard forced response, and also because of the type of the nonlinearity considered (discontinuity). Nevertheless, future developments should investigate the applicability of NNM for optimal sensor placement in nonlinear structures. 
The present work aims to automatically choose the location of sensors in a nonlinear dynamical structure under a given operational condition. It uses the EIDV procedure, but the modes considered in Fisher information matrix are the proper orthogonal modes of the structure obtained applying Karhunen-Loève decomposition (KLD) [17], also called proper orthogonal decomposition (POD). This is a stochastic tool that showed to be well suited for deterministic systems. The proper orthogonal modes (POM) provide the coherent structures underneath the response of the dynamical system. We will call this strategy effective independence proper orthogonal modes (EIPOM).

There are two ways to compute the POMs: (1) with numerical data or (2) with experimental data. If a computational model of the nonlinear system is available, it can be excited in the operational condition to compute the displacement response. Only the locations that can be measured are retained for the computation of the POMs, and then the EIDV procedure can iteratively dismiss the remaining locations that are not relevant. On the other hand, if measurements are taken for the structure in operation (ideally in every possible measurement location), the displacement field obtained by the measurements can be used to compute the POMs, and then the EIDV procedure can iteratively dismiss locations that are not relevant.

The strategy proposed in this work is expected to be well suited to any type of nonlinear structure that needs to be monitored, such as cars, airplanes, bridges, and platforms. It suffices to (1) know the operational condition and to have a calibrated computational model or (2) have experimental data of the structure in the operational condition. A simple cantilever beam with local nonlinearities (impacts) is used to exemplify the EIPOM strategy.

This paper is organized as follows. Section 2 presents the equations of the computational models (linear and nonlinear) used in the analysis. Section 3 develops the EIPOM procedure. Finally, the numerical results are presented in Section 4 and the concluding remarks are made in Section 5.

\section{Computational Model}

Consider a structure that has been discretized by means of the finite element method, with discretized system, given by

$$
[M] \ddot{\mathbf{u}}(t)+[C] \dot{\mathbf{u}}(t)+[K] \mathbf{u}(t)=\mathbf{f}(t),
$$

where $[M] \in \mathbb{R}^{m \times m}$ is the positive-definite mass matrix, $[C] \in \mathbb{R}^{m \times m}$ is the proportional damping matrix that added a posteriori to the model $([C]=\alpha[M]+\beta[K]),[K] \in \mathbb{R}^{m \times m}$ is the positive-definite bending matrix, $\mathbf{u}(t) \in \mathbb{R}^{m}$ is the response vector, and $\mathbf{f}(t) \in \mathbb{R}^{m}$ is the force vector. This system is linear; the nonlinearity is going to be added to the system further in this section.

To speed up the computation, a reduced-order model [18, $19]$ is going to be constructed for the system. The following generalized eigenvalue problem is solved to compute the reduction basis, which is composed by the normal modes of the linear structure

$$
\left(-\omega^{2}[M]+[K]\right) \phi=\mathbf{0},
$$

where $\omega_{i}$ is the $i$ th natural frequency and $\phi_{i}$ is the $i$ th normal mode. Defining matrix $[\Phi]=\left[\begin{array}{llll}\phi_{1} & \phi_{2} & \cdots & \phi_{n}\end{array}\right]$ and writing the displacement as $\mathbf{u}(t)=[\Phi] \mathbf{q}(t)$, the reduced-order system is given by

$$
\left[M_{r}\right] \ddot{\mathbf{q}}(t)+\left[C_{r}\right] \dot{\mathbf{q}}(t)+\left[K_{r}\right] \mathbf{q}(t)=[\Phi]^{T} \mathbf{f}(t),
$$

where the reduced matrices are given by $\left[M_{r}\right]=$ $[\Phi]^{T}[M][\Phi],\left[C_{r}\right]=[\Phi]^{T}[C][\Phi]$, and $\left[K_{r}\right]=[\Phi]^{T}[K][\Phi]$, with $\left[M_{r}\right]=\delta_{i j},\left[K_{r}\right]=\delta_{i j} \omega_{i}^{2}$ and $\left[C_{r}\right]=2 \omega_{i} \xi_{i} \delta_{i j}$, in which $\xi_{i}=\alpha /\left(2 \omega_{i}\right)+\beta \omega_{i} / 2$ is the damping factor and $\delta$ is the Kronecker delta.

Local nonlinearities represented by impacts are taken into account. The impact between the structure and the barrier is modeled with a linear spring with constant $k_{\mathrm{sh}}$ :

$$
\mathbf{f}_{i}(x, t)= \begin{cases}-k_{\mathrm{sh}} u\left(x_{i}, t\right) \delta_{d}\left(x-x_{i}\right) & \text { if } u\left(x_{i}, t\right)>\text { gap } \\ 0 & \text { if } u\left(x_{i}, t\right) \leq \text { gap }\end{cases}
$$

where gap is the distance between the structure and the barrier, $u\left(x_{i}, t\right)$ is the displacement at the impact locations $(i=\{1,2, \ldots\})$, and $\delta_{d}$ is the Dirac delta. Hence, the nonlinear system of equations is given by

$$
\begin{aligned}
{\left[M_{r}\right] } & \ddot{\mathbf{q}}(t)+\left[C_{r}\right] \dot{\mathbf{q}}(t)+\left[K_{r}\right] \mathbf{q}(t) \\
& =[\Phi]^{T}\left(\mathbf{f}(t)+\mathbf{f}_{\mathrm{NL}}([\Phi] \mathbf{q}(t))\right),
\end{aligned}
$$

where $\mathbf{f}_{\mathrm{NL}}$ is the nonlinear force vector related to the impacts.

\section{Effective Independence Proper Orthogonal Modes}

This section describes the proposed method called effective independence proper orthogonal modes (EIPOM), where POMs are used in the effective independence strategy. The EIVD strategy is very easy to implement, it is not computationally expensive, and it gives some physical insight to the selection of sensor locations. This method was developed by Kammer [11] as a procedure to validate a large space structure finite element model. The idea is to maximize the determinant of Fisher information matrix:

$$
[\mathrm{FIM}]=\Psi^{T} \Psi,
$$

where $\Psi$ is the reduced modal matrix composed by the selected normal modes of the linear structure without the degrees of freedom that cannot be measured (rotation, for instance). If the modes are independent, [FIM] is invertible. The procedure is based on the observation of the diagonal of matrix $[E][9]$ :

$$
[E]=\Psi[\mathrm{FIM}]^{-1} \Psi^{T} .
$$

The diagonal elements of $[E], \operatorname{diag}([E])$, represent the fractional distribution of each measurement location to the rank of $[E]$. The degrees of freedom with least contribution are removed, and $\Psi$ is updated. This process eliminates locations iteratively. It should be noticed that maximizing $\operatorname{det}([\mathrm{FIM}])$ 
is the same as maximizing independent information, and it tends to minimize the covariance matrix of the estimate errors [11].

Since we are dealing with a nonlinear structure, an extension of the above procedure is proposed because it does not make much sense to talk about normal modes any more. Karhunen-Loève decomposition (KLD) [17] is also known as proper orthogonal decomposition (POD) and is an extension of the principal component analysis (PCA) [20] to infinity dimension. POD has many applications, such as the construction of reduced-order models for dynamical systems [18, 21]. It was first applied to mechanical systems for the research of coherent structures in turbulent flows [22]. We will use this methodology to compute the POMs, also called coherent structures or Karhunen-Loève basis (KL-basis).

When the system is nonlinear there are no normal modes related to it. However, we can construct the POMs, which are able to retain the important characteristics of a dynamical response through experimental or numerical data.

It is assumed that a zero mean stationary second order random field $\left\{V(t, \mathbf{x}),(t, \mathbf{x}) \in \mathbb{R} \times \mathbb{R}^{3}\right\}$ is continuous in mean square. This process is obtained by subtracting the mean of the displacement random field of the structure $\{U(t, \mathbf{x})$, $\left.(t, \mathbf{x}) \in \mathbb{R} \times \mathbb{R}^{3}\right\}$. Therefore, we assume that the displacement field is a stationary second order random field, which might be a strong assumption. However, we are considering the steady state response of a system in a given operational condition; thus, this assumption is likely to work well. The autocorrelation function of $V(t, \mathbf{x})$ is given by

$$
R\left(t_{1}, \mathbf{x}_{1} ; t_{2}, \mathbf{x}_{2}\right)=\mathbb{E}\left\{\left(V\left(t_{1}, \mathbf{x}_{1}\right)\right)\left(V\left(t_{2}, \mathbf{x}_{2}\right)\right)\right\},
$$

where second order stationarity means $R\left(t_{1}, \mathbf{x}_{1} ; t_{2}, \mathbf{x}_{2}\right)=$ $R\left(t_{2}-t_{1}, \mathbf{x}_{1}, \mathbf{x}_{2}\right)$, and $\mathbb{E}\{\cdot\}$ is the expectation operator. The POMs $\phi_{k}^{\mathrm{POM}}$ are obtained by solving the following eigenvalue problem:

$$
\int_{\mathbb{R}^{3}} R\left(0, \mathbf{x}, \mathbf{x}^{\prime}\right) \phi_{k}^{\mathrm{POM}}(\mathbf{x}) d \mathbf{x}=\lambda_{k} \phi_{k}^{\mathrm{POM}}(\mathbf{x}),
$$

where the $\lambda$ s are ordered from high to low and are normalized by dividing each $\lambda$ by the sum of $\lambda \mathrm{s}$, such that the sum of the normalized eigenvalues is one. They are a measure of the energy of each POM, which means that the POMs related to high $\lambda$ s are more important for the dynamics.

Another useful assumption is to consider the process $V(t, \mathbf{x})$ ergodic, such that only one dynamic response is needed to construct the autocorrelation function.

\section{Application}

For its simplicity and to focus on the strategy of selecting sensor locations, a cantilever Timoshenko beam is considered in the analysis. Figure 1 shows the nonlinear system analyzed, where the nonlinearity comes from impacts. It consists in a cantilever beam harmonic excited at $x=L$ and with two barriers located at $\{0.23 L, 0.48 L\}$. This system is inspired by an overhung rotor-bearing system $[23,24]$.

The parameter values used in the numerical analysis are $\rho=7850 \mathrm{~kg} / \mathrm{m}^{3}, E=200 \mathrm{GPa}, G=81 \mathrm{GPa}, \xi_{i}=5 \%$,

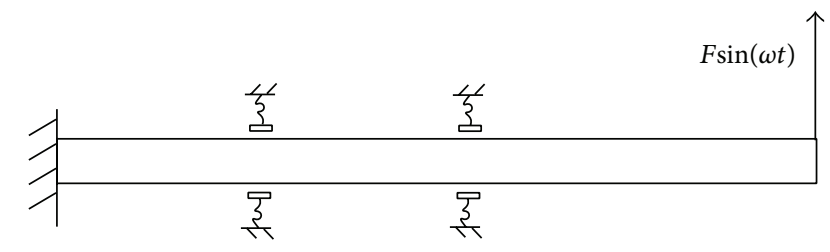

FIGURE 1: Nonlinear system with linear impact forces at two points and harmonic excitation at $x=L$.

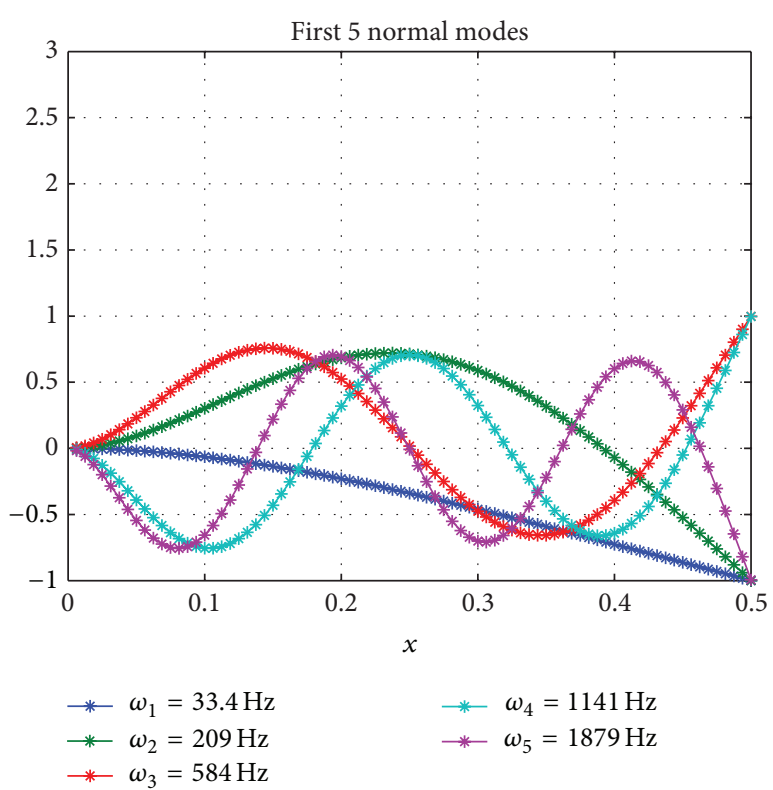

FIGURE 2: First five normal modes of a cantilever beam with the corresponding natural frequencies.

$L=0.5 \mathrm{~m}, A=1 \times 10^{-4} \mathrm{~m}^{2}, I=8.3 \times 10^{-10} \mathrm{~m}^{4}, k_{\mathrm{sh}}=0.87$, gap $=1 \times 10^{-4} \mathrm{~m}, k_{\mathrm{sh}}=1 \times 10^{6} \mathrm{~N} / \mathrm{m}, F=20 \mathrm{~N}$, and $\omega_{f}=60 \mathrm{~Hz}$. The beam is discretized in 40 finite elements and 10 normal modes are sufficient for the dynamic analysis. The system is integrated using the explicit Runge Kutta scheme of 4 th order with adaptive time steps, and zero initial conditions were considered. For the construction of the POMs, the time range considered was $[0.25 \mathrm{~s}, 0.5 \mathrm{~s}]$ to discard transient response, time step of $1.7 \times 10^{-4} \mathrm{~s}$, and spatial discretization of $0.0125 \mathrm{~m}$.

4.1. Linear System. This section analyzes a linear cantilever beam. The goal is to show that the placement of sensors is different if one is interested in (a) obtaining the dynamics characteristics of a system or (b) monitoring a system with a given operational condition. It should not be a surprise, since we would expect to obtain different sensor locations if different modes are considered.

To validate the EIDV procedure, the individual normal modes of a clamped and simply supported beam were evaluated. As expected, the optimal sensor locations for the individual normal modes coincided with the maximum amplitude values of each mode. 


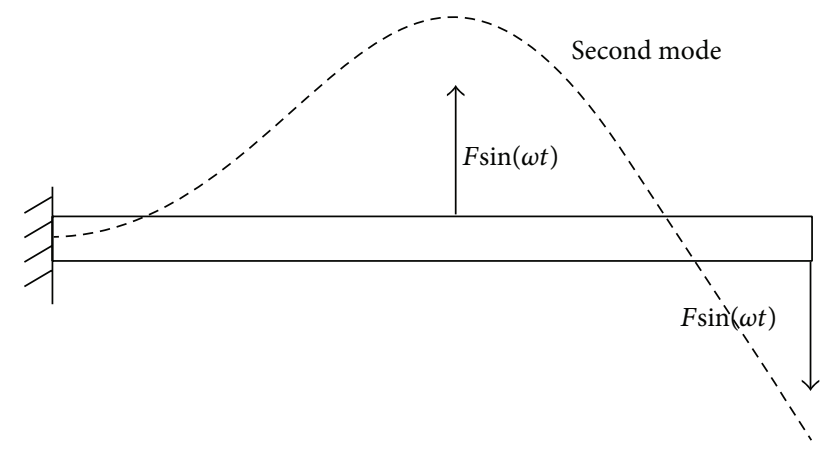

FIGURE 3: Clamped beam with two external forces together with the second mode. Even if the excitation frequency is lower than the 1st natural frequency of the system, depending on the force locations, other modes might play a role in the dynamics. For the configuration in the figure, the second mode will be important.

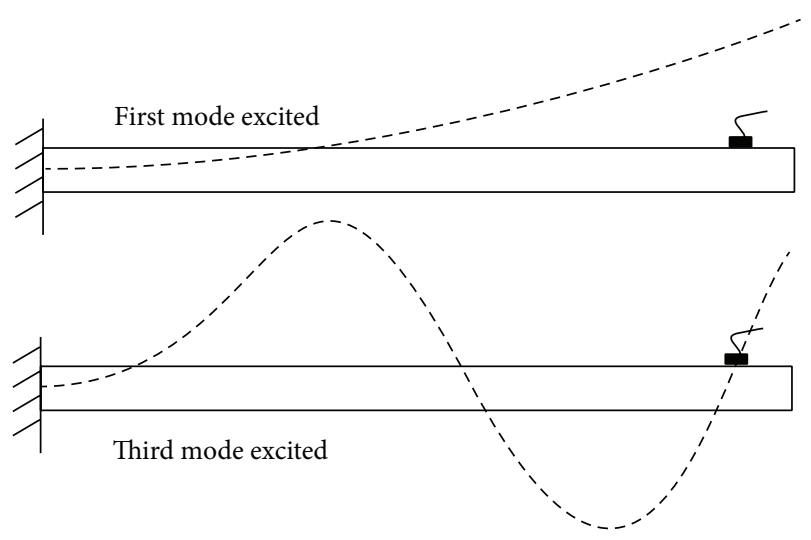

FIGURE 4: First and third modes of a clamped beam. Optimal measurement location depends on the excitation of the structure. Figure shows that the sensor location is good if the first mode is predominant in the dynamics but poor if the third mode dominates the dynamics.

Figure 2 shows the first five normal modes of a cantilever beam with the corresponding natural frequencies. Figure 3 shows that even if the frequency of excitation is lower than the first mode, depending on the configuration of the applied forces, higher modes can be important in the dynamical response of the structure, which means that one should take into account a typical operational condition. For the configuration in the figure, the second mode will play an important role. Finally, Figure 4 shows that a specific sensor location might be good or bad, depending on the mode excited. In the figure, the sensor location is good if the first mode is excited and bad if the third mode is excited.

To analyze some numerical results, let us choose the measurement locations of 4 sensors for two different operational conditions. In the first operational condition, four modes are important in the dynamics. On the other hand, in the second operational condition, only two modes are important; see Figure 5. If modes from one to four are considered, the resulting locations are $\{0.26 L, 0.51 L, 0.78 L, L\}$. On the other hand, if only modes three and four are considered,

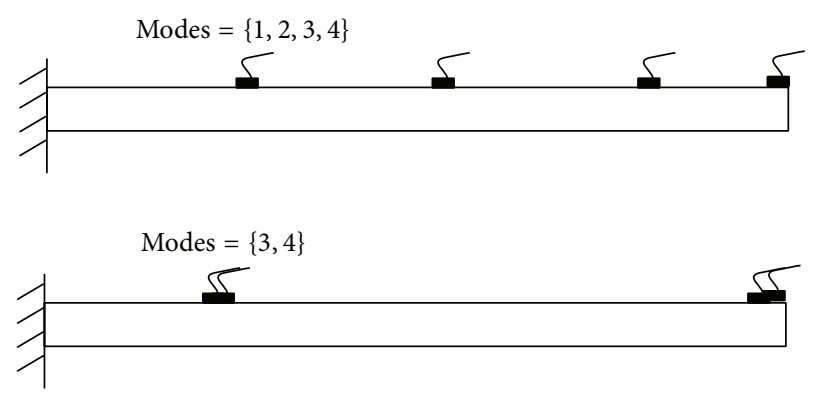

FIGURE 5: Different result for measurement locations of 4 sensors (a) if modes 1 to 4 are important and (b) if only modes 3 and 4 are important for the dynamic response.

the resulting locations are $\{0.23 L, 0.24 L, 0.99 L, L\}$. Of course, in this last case only two sensors are needed.

If the system is linear and if one can filter the relevant modes contributing to the dynamics of a given operational condition; then, the EIDV procedure could be applied with the selected normal modes.

4.2. Nonlinear System. Now the nonlinear system is analyzed. The nonlinear system is harmonically excited at $x=L$ and two impact locations are considered, as shown in Figure 1. To construct the POMs, a sample of the steady state response of the numerical model is obtained, and the direct KarhunenLoève decomposition is applied.

Figure 6(a) shows the first two POMs of the structure, responsible for $99.99 \%$ of the energy $\left(\lambda_{1}=0.9978\right.$ and $\lambda_{2}=$ $0.0021)$. It also shows the impact locations and the location of the 4 sensors obtained using EIPOM $\{0.63 L, 0.65 L, 0.98 L, L\}$.

Figure 6(b) compares these two modes with the first two normal modes of the structure. If one uses the first 2 normal modes of the structure to place the sensors, the result would be quite different $\{0.54 L, 0.55 L, 0.99 L, L\}$. Finally, since all of the 10 normal modes play a role in the present nonlinear dynamics, one could try to use all of them in the EIVD strategy, but the results do not get better $\{0.20 \mathrm{~L}, 0.30 \mathrm{~L}, 0.80 \mathrm{~L}$, $0.91 L\}$.

A limitation of EIPOM strategy is that the POMs are different for different operational conditions. Figure 7 shows how different the two first modes are for two values of the impact stiffness parameter. Two possible solutions for this problem are given. (1) Compute the set of POMs separately for each $i$ th operational condition, $\left[\Psi_{i}\right]$, and place the sensors accordingly to the locations obtained for each $i$ th operational condition depending on the current operational condition; this can be done by changing the sensor locations or putting sensors for all the important locations. (2) If there are many operational conditions or if it is not possible to place/change all the necessary sensors, an extended matrix can be constructed with the modes obtained for each $i$ th operational condition, $\left[\Psi_{e}\right]=\left[\left[\Psi_{1}\right]\left[\Psi_{2}\right] \cdots\left[\Psi_{n_{\mathrm{op}}}\right]\right]$ (where $n_{\mathrm{op}}$ is the number of operational conditions included in the analysis), and $\left[\Psi_{e}\right]$ can be used in the EIDV procedure. However, if an operational condition is missing, the locations obtained with EIPOM will not be optimal (or suboptimal). 


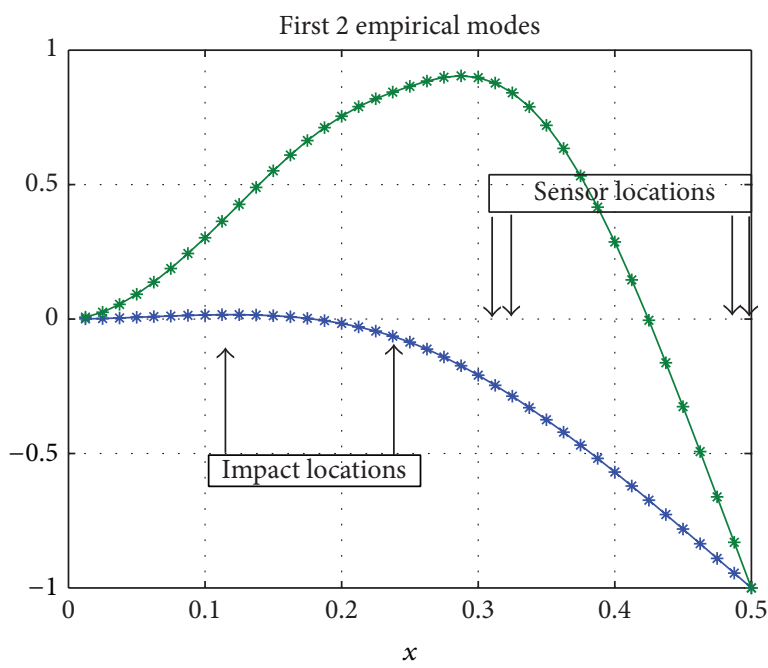

(a)

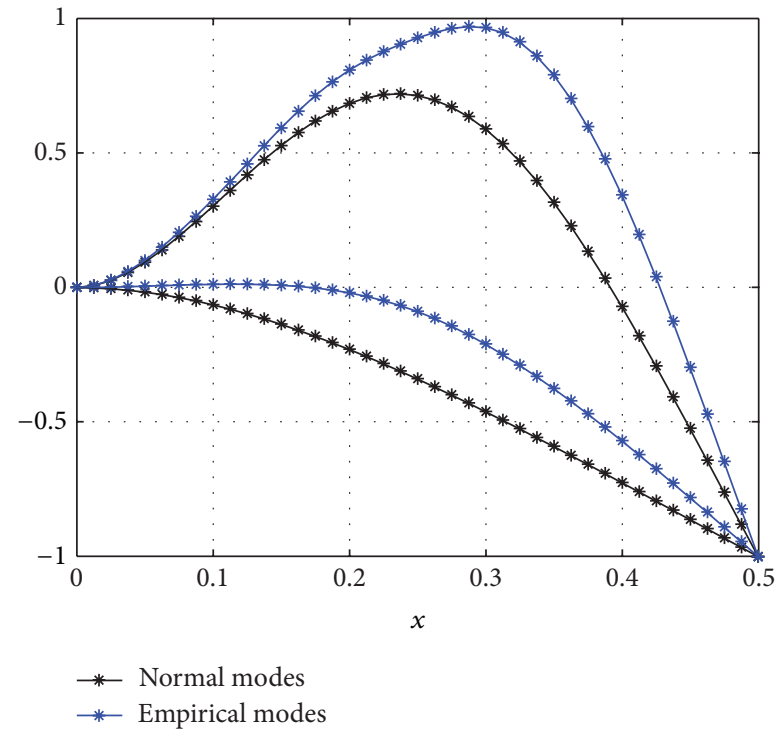

(b)

FIGURE 6: (a) First two proper orthogonal modes of the nonlinear system with the corresponding impact locations; (b) proper orthogonal $\times$ normal modes.

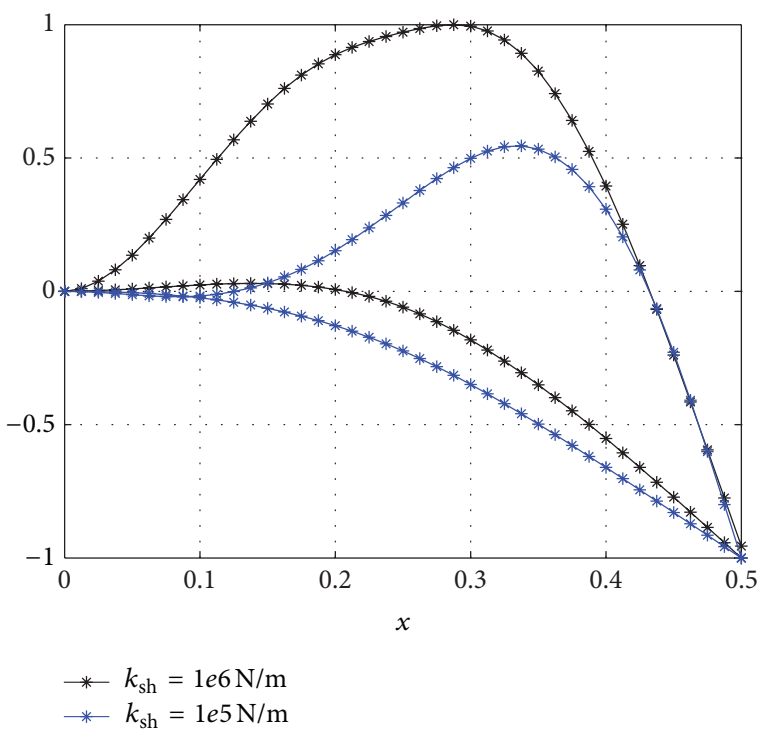

FIGURE 7: First two proper orthogonal modes of the nonlinear system with different $k_{\mathrm{sh}}$ values.

\section{Concluding Remarks}

This paper proposes to use together the EIDV and the POMs to choose the location of sensors to monitor a nonlinear dynamical system, in a way that a nonlinear structure/equipment can be monitored effectively. The idea is simple and effective. Instead of using the normal modes in the EIDV procedure, one should use the POMs. Hence, the locations chosen are maximizing the determinant of Fisher information matrix, maximizing independent information, and it tends to minimize the covariance matrix of the estimate errors. The proposed procedure is not effective if the operational condition changes. In this case, two alternatives are proposed: (1) a set of POMs should be constructed for each specific operational condition or (2) all the operational conditions should be used together in order to construct the POMs.

Future investigations should be made to analyze the use of POMs in other techniques that use the effective independence strategy, such as EMRO [13] and EOT [14], when the system is nonlinear.

\section{Conflict of Interests}

The author declares that there is no conflict of interests regarding the publication of this paper.

\section{Acknowledgments}

The author is grateful to the financial support from the Brazilian agencies: CAPES, CNPq, and FAPERJ.

\section{References}

[1] G. L. C. M. Abreu, J. F. Ribeiro, and V. Steffen Jr., "Experiments on optimal vibration control of a flexible beam containing piezoelectric sensors and actuators," Shock and Vibration, vol. 10, no. 5-6, pp. 283-300, 2003.

[2] C. T. Chen, Applied System Theory and Design, Harcourt Brace College, Fort Worth, Tex, USA, 1984.

[3] K.-V. Yuen, L. S. Katafygiotis, C. Papadimitriou, and N. C. Mickleborough, "Optimal sensor placement methodology for identification with unmeasured excitation," Journal of Dynamic Systems, Measurement and Control-Transactions of the ASME, vol. 123, no. 4, pp. 677-686, 2001. 
[4] N. Debnath, A. Dutta, and S. K. Deb, "Placement of sensors in operational modal analysis for truss bridges," Mechanical Systems and Signal Processing, vol. 31, 196216 pages, 2012.

[5] F. E. Udwadia and J. A. Garba, "Optimal sensor locations for structural identification," in Proceedings of the JPL Workgroup on Identification and Control of Flexible Space Structures, pp. 247-261, San Diego, Calif, USA, 1985.

[6] R. E. Miller, "Optimal sensor placement via Gaussian quadrature," Applied Mathematics and Computation, vol. 97, no. 1, pp. 71-97, 1998

[7] K. Hiramoto, H. Doki, and G. Obinata, "Optimal sensor/actuator placement for active vibration control using explicit solution of algebraic Riccati equation," Journal of Sound and Vibration, vol. 229, no. 5, pp. 1057-1075, 2000.

[8] R. J. Guyan, "Reduction of stiffness and mass matrices," AIAA Journal, vol. 3, no. 2, article 380, 1965.

[9] J. E. T. Penny, M. I. Friswell, and S. D. Garvey, "Automatic choice of measurement locations for dynamic testing," AIAA Journal, vol. 32, no. 2, pp. 407-414, 1994.

[10] Z. H. Qureshi, T. S. Ng, and G. C. Goodwin, "Optimum experimental design for identification of distributed parameter systems," International Journal of Control, vol. 31, no. 1, pp. 21-29, 1980.

[11] D. C. Kammer, "Sensor placement for on-orbit modal identification and correlation of large space structures," Journal of Guidance, Control, and Dynamics, vol. 14, no. 2, pp. 251-259, 1991.

[12] D. E. Holland, M. P. Castanier, S. L. Ceccio, B. I. Epureanu, and S. Filippi, "Testing and calibration procedures for mistuning identification and traveling wave excitation of blisks," Journal of Engineering for Gas Turbines and Power, vol. 132, no. 4, Article ID 042502, 2010.

[13] F. M. Hemez and C. Farhat, "An energy based optimum sensor placement criterion and its application to structure damage detection," in Proceedings of the 12th International Modal Analysis Conference, pp. 1568-1575, Society of Experimental Mechanics, Honolulu, Hawaii, USA, 1994.

[14] G. Heo, M. L. Wang, and D. Satpathi, "Optimal transducer placement for health monitoring of long span bridge," Soil Dynamics and Earthquake Engineering, vol. 16, no. 7-8, pp. 495502, 1997.

[15] A. H. Nayfeh, Nonlinear Interactions, Wiley-Interscience, New York, NY, USA, 2000.

[16] G. Kerschen, M. Peeters, J. C. Golinval, and A. F. Vakakis, "Nonlinear normal modes, part I: a useful framework for the structural dynamicist," Mechanical Systems and Signal Processing, vol. 23, no. 1, pp. 170-194, 2009.

[17] M. Loève, Probability Theory. I, Springer, New York, NY, USA, Fourth edition, 1977.

[18] G. Kerschen, J.-C. Golinval, A. F. Vakakis, and L. A. Bergman, "The method of proper orthogonal decomposition for dynamical characterization and order reduction of mechanical systems: an overview," Nonlinear Dynamics, vol. 41, no. 1-3, pp. 147-169, 2005.

[19] T. G. Ritto, F. S. Buezas, and R. Sampaio, "A new measure of efficiency for model reduction: application to a vibroimpact system," Journal of Sound and Vibration, vol. 220, no. 9, pp. 19771984, 2011.

[20] I. T. Jolliffe, Principal component analysis, Springer, New York, NY, USA, 1986.
[21] S. Bellizzi and R. Sampaio, "POMs analysis of randomly vibrating systems obtained from Karhunen-Loève expansion," Journal of Sound and Vibration, vol. 297, no. 3-5, pp. 774-793, 2006.

[22] J. L. Lumley, Stochastic Tools in Turbulence, Academic Press, New York, NY, USA, 1970.

[23] M. F. A. Azeez and A. F. Vakakis, "Numerical and experimental analysis of a continuous overhung rotor undergoing vibroimpacts," International Journal of Non-Linear Mechanics, vol. 34, no. 3, pp. 415-435, 1998.

[24] T. G. Ritto and R. Sampaio, "The effects of unbalance and clearance on the bearings of an overhung rotor," in Proceedings of the 18th COBEM, Ouro Preto, Brazil, 2005. 

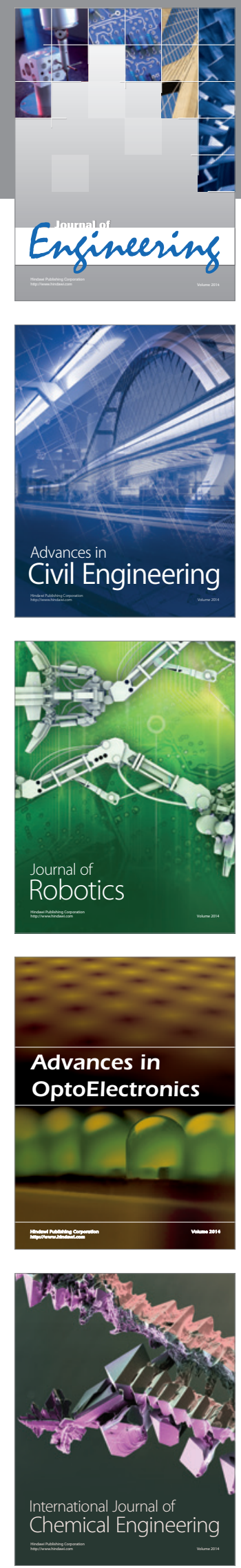

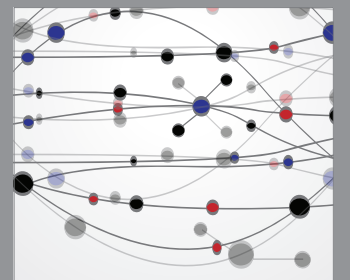

The Scientific World Journal
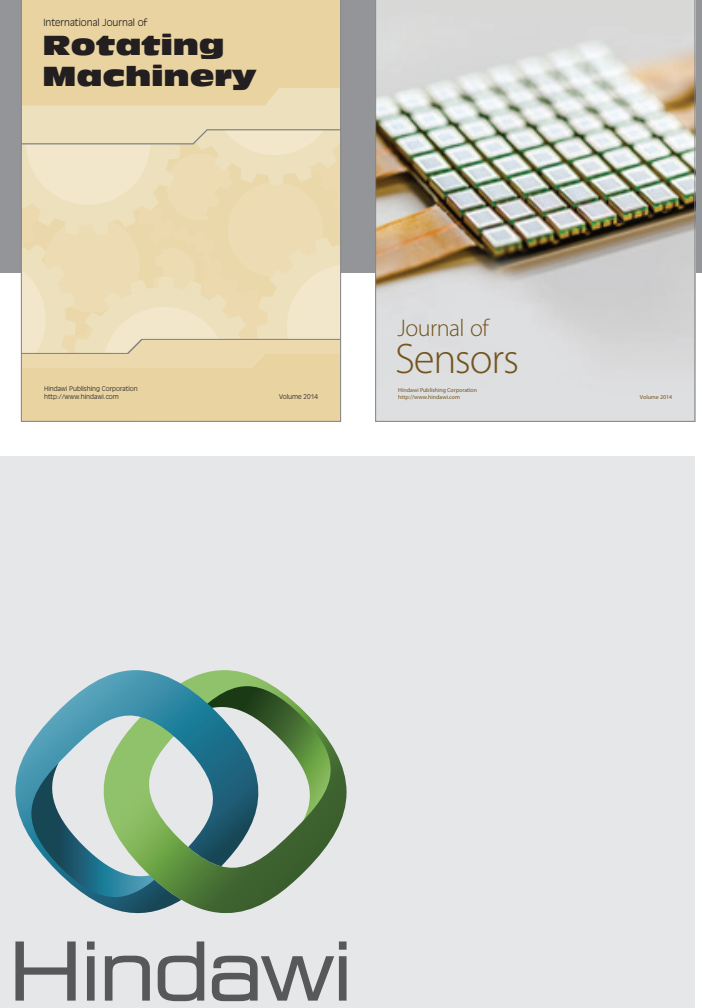

Submit your manuscripts at http://www.hindawi.com
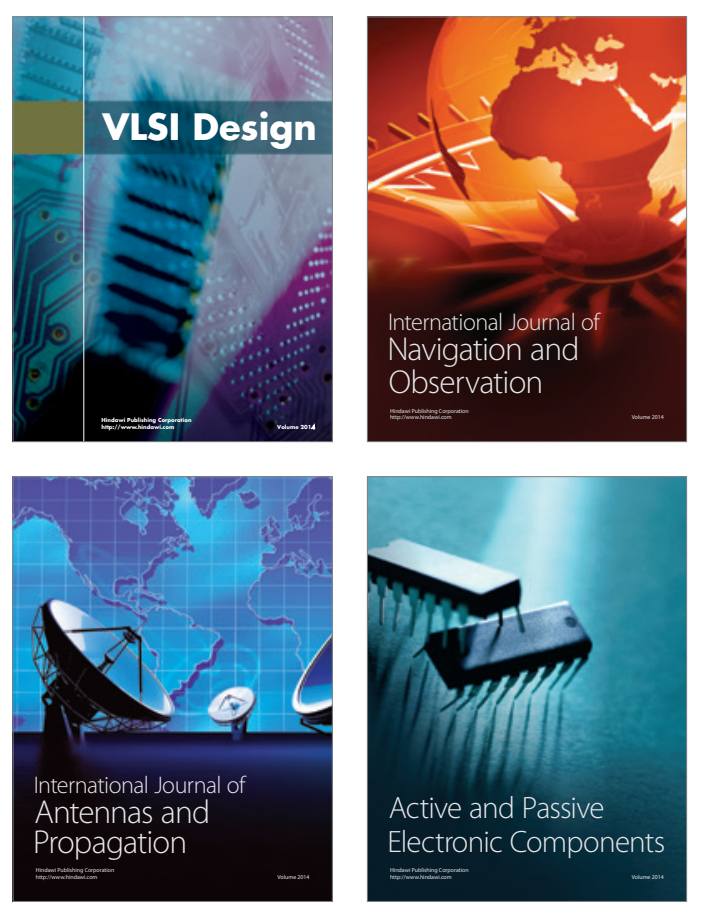
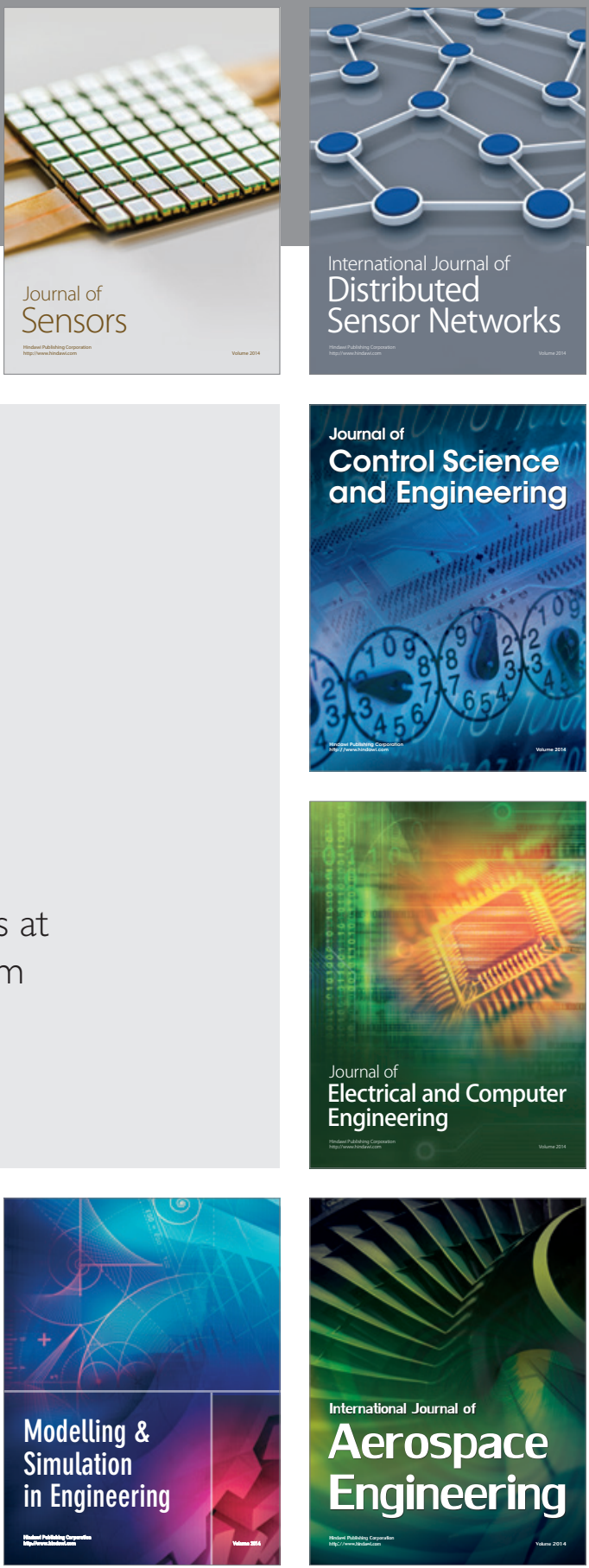

Journal of

Control Science

and Engineering
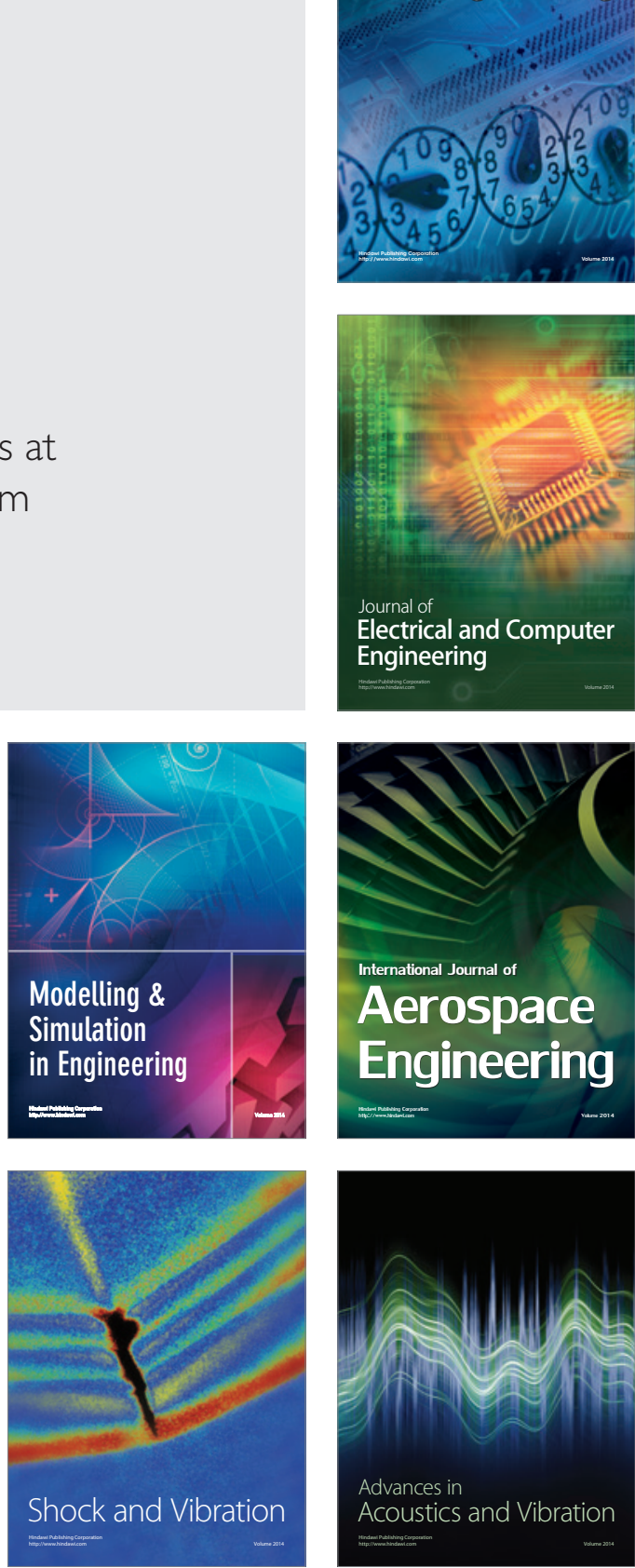